\title{
RECENT DEVELOPMENTS IN NON-PERTURBATIVE QUANTUM FIELD THEORY
}

\author{
Sergio FERRARA \\ Theoretical Physics Division, CERN, 1211 Geneva 23, Switzerland
}

\begin{abstract}
We report on recent advances in the understanding of non-perturbative phenomena in the quantum theory of fields and strings.
\end{abstract}

\section{Introduction}

In the last couple of years we have been witnessing a series of impressive results in the framework of two different but related types of quantum theories: supersymmetric Yang-Mills theories in four dimensions 1 and superstringse. They have in common space-time supersymmetry. They also share the property that a formal perturbative expansion can be defined for these theories, and "renormalization" can be used in order to extract finite answers from any perturbative calculation.

These theories enjoy non-renormalization theorems 3 depending on the degree of supersymmetry of the vacuum around which we define the quantum perturbative series.

Let us recall the spin content of the light states: the gauge theory includes massless quanta with spin $0,1 / 2,1$; superstrings include massless quanta with spin $0,1 / 2,1,3 / 2$ and 2 ; in a suitable limit ( $\alpha^{\prime} \rightarrow 0$, gauge-coupling fixed) superstrings reproduce ordinary gauge theories.

Exact non-perturbative results of SQCD have been obtained. Seiberg and Witten 1 suggested an exact expression for the low-energy effective action for the Coulomb phase of an $N=2 \mathrm{SU}(2) \mathrm{SYM}$ theory, which may be regarded as an extension of the Georgi-Glashow SU(2) gauge theory.

Electric-magnetic duality plays a crucial role to solve the theory, to compute strong coupling phases of this theory (where massless monopoles and dyons appear), and to prove colour confinement through a magnetic Higgs mechanism with a monopole condensation (analogue to Meissner effects in superconductors).

The solution of the problem is possible thanks to non-renormalization theorems, making the complete perturbative computation affordable.

The non-perturbative part, conjecturally due to processes from multi-instanton transitions, is obtained from a mathematical hypothesis that identifies electric and magnetic massive states with windings $(n, m)$ of a genus 1 (torus) elliptic Riemann surface (genus $n$ for an $\mathrm{SU}(n)$ gaugetheory as shown by Klemm, Lerche, Theisen and Yankielowicz and by Argyres and Faraggi 5). Two topologically different cycles correspond to electric and magnetic charges. Quantum massive BPS states $\psi(n, m)$ correspond to distinct topological configurations $(n, m)$ of the elliptic surface, in particular $\psi(1,0)=W$-boson, $\psi(0,1)=$ monopole, $\psi(-1,+1)=$ dyon.

\section{Electric-Magnetic Duality and Super- symmetry}

Let us recall the duality of Maxwell equations

$$
\begin{gathered}
\nabla \cdot(E+i B)=\rho_{e}+i \rho_{m}=\rho \\
\nabla \wedge(E+i B)-i \frac{\partial}{\partial t}(E+i B)=J_{e}+i J_{m}=J \\
L=\operatorname{Re}(E+i B) \cdot(E+i B)=E^{2}-B^{2} .
\end{gathered}
$$

Here $E, B$ are the electric and magnetic fields; $\rho_{e}\left(\rho_{m}\right), J_{e}\left(J_{m}\right)$ denote electric (magnetic) charge density and current, respectively. A topological term $E \cdot B$ may eventually be added to $L$. The physical observables such as the energy density $(E+i B) \cdot(E-i B)=E^{2}+B^{2}$, and the momentum density $(E+i B) \wedge(E-i B)$ are invariant under (continuous) $\mathrm{U}(1)$ duality rotations:

$(E+i B) \rightarrow e^{i \varphi}(E+i B), \quad \rho \rightarrow e^{i \varphi} \rho, \quad J \rightarrow e^{i \varphi} J$.

In particular the $Z_{2}$ symmetry, which is the remnant of $U(1)$, acting on discrete charged states, exchanges electric with magnetic fields $E \rightarrow$ $B$ and $B \rightarrow-E$, and electric and magnetic charges $q \rightarrow g, g \rightarrow-q$, accordingly.

The simultaneous occurrence of electric and magnetic sources implies a charge quantization, which reads:

$$
q g=2 \pi k
$$

(Dirac 1931) ${ }^{6}$ (for monopoles) 
and

$$
q_{1} g_{2}-q_{2} g_{1}=2 \pi k
$$

(Schwinger, Zwanziger 1968) ${ }^{7}$ (for dyons).

In the Coulomb phase the Georgi-Glashow SU(2) gauge theory has a monopole with mass ('t Hooft, Polyakov 1974) 6 :

$$
M_{\text {monopole }} \geq 1 / \lambda\langle\phi\rangle
$$

(Bogomolny bound, 1975) ${ }^{9}$

while the classical vector boson mass is $M_{W}$ = $\lambda\langle\phi\rangle$. In the Prasad-Sommerfeld (1976) limit 10 (supersymmetry) $M_{\text {monopole }}=1 / \lambda\langle\phi\rangle$ satisfies the duality conjecture (Montonen, Olive 1977) 11 :

$$
M^{2}(q, g)=M^{2}\left(q^{2}+g^{2}\right)=\langle\phi\rangle^{2}\left(q^{2}+g^{2}\right) .
$$

This generalizes when a topological term $\theta E \cdot B$ is included by defining a complex parameter $\tau=$ $\theta+i / \lambda^{2}$ and then writing:

$$
M^{2}(\phi, \tau, n, m)=\frac{\left|\phi^{2}\right|}{l m \tau}|n+\tau m|^{2}
$$

invariant under $S L(2, Z)$ :

$$
\begin{aligned}
Z_{2} & : \quad \tau \rightarrow \frac{-1}{\tau}, \quad n \rightarrow m, \quad m \rightarrow-n \\
\theta-\text { shift } & : \quad \tau \rightarrow \tau+1, \quad n \rightarrow n-m, \quad m \rightarrow m .
\end{aligned}
$$

This means that the dual theory obtained by a $Z_{2}$ symmetry $E \rightarrow B, B \rightarrow-E$ has $\tau_{d}=-1 / \tau, n_{D}=$ $m, m_{D}=-n$. The $N=4$ supersymmetric YangMills theory realizes the Montonen-Olive duality conjecture 11 The theory has an exact $\mathrm{SL}(2, Z)$ symmetry 12 , which is possible in virtue of a vanishing $\beta$ function, in the full quantum theory. Electric states are fundamental, while magnetic states are solitons in the theory $T$, but their role is reversed in the dual theory $T_{D}$.

Seiberg and Witten $t$ extended the duality to $N=2$, SYM quantum field theories undergoing renormalization $(\beta \neq 0)$, which gives corrections to a 'holomorphic prepotential', $F(\phi)$; this is the appropriate tool to build up $N=2$ effective actions. The BPS states (which lie in hypermultiplets have mass $M(\phi, n, m, \lambda) \propto\left|\phi n+F_{\phi} m\right|$ where $33(\phi)=(i / 2 \pi) \phi^{2} \ln \left(\phi^{2} / \lambda^{2}\right)+\ldots$ (the dots denote the non-perturbative contributions).

They also extended the duality conjecture. This came by identifying the pair $\left(\phi, F_{\phi}\right)$ with the periods of an hyper-elliptic surface, which allows us to give a closed expansion for $F(\phi)$. As a result of this at strong coupling $\phi^{2} / \lambda^{2}= \pm 1$, one gets a massless monopole $(0,1)$ and a dyon $(-1,+1)$.

The dual (U(1) magnetic) theory is weakly coupled in the strong coupling of the electric theory and describes a magneto-dynamic of a charged monopole. In the weakly coupled magnetic Higgs phase, monopole condensation describes confinement of the original (strongly coupled) electric theory. It is worth mentioning that, for BPS states, their mass appears in the central extension of the supersymmetry algebra (HaagLopuszanski-Sohniys) 14 and this allows one, using supersymmetry 15 , to compute their mass in terms of the low-energy data. The duality has been further extended to $N=1$ super-Yang-Mills theories 16, in particular to SQCD with colour group $\mathrm{SU}\left(N_{c}\right)$ and $N_{f}$ flavours. This theory has an anomaly-free global symmetry:

$$
S U_{L}\left(N_{f}\right) \times S U_{R}\left(N_{f}\right) \times U(1)_{B} \times U(1)_{R} .
$$

Seiberg suggested that there is a non-Abelian Coulomb phase for $3 / 2 N_{c}<N_{f}<3 N_{c}$. At the non-trivial infra-red fixed point, the theory of quarks and gluons has a dual description in terms of an interacting conformal invariant theory with magnetic gauge group $S U\left(N_{f}-N_{c}\right)$ and $N_{f}$ flavours. Quarks and gluons are solitons in the dual picture.

\section{Supergravity, Strings and $M$-Theory}

Duality symmetries in the context of supergrayity theories 17 , further extended to superstrings 18 , allow us to prove exact equivalences of different string theories 19 20, to obtain a dynamical understanding of the Seiberg-Witten conjecture in the point-particles limit 223 and finally to possibly merge these theories in the context of $M$ theory, a supposedly existine quantum theory of membranes and five-branes2425, whpse low-energy effective action is $11 \mathrm{D}$ supergravity 26 .

There are five known types of superstring theories in 10 dimensionst:

\begin{tabular}{l|l} 
Type & Gauge group \\
\hline Type I & $\mathrm{SO}(32)$ \\
Heterotic & $\mathrm{SO}(32), E_{8} \times E_{8}$ \\
Type IIA & $\mathrm{U}(1)$ \\
Type IIB & None
\end{tabular}


The first three have $N=1$ supersymmetry, while the last two have $N=2$, non-chiral type IIA and chiral IIB. There is also a conjectured $M$-theory in 11 dimensions 21 (no gauge group). Upon reduction on a circle, this is equivalent to type IIA, at the non-perturbative level. A further speculative theory may exist in twelve dimensions, which gives, upon reduction on a two-torus, the type IIB theory 27.

The previous theories, and their compactification to lower dimensions, reduce at low energies to supergravity theories in diverse dimensions 28 with underlying supersymmetry algebras as classified by Nahm 29. In the highest and lowest dimensions of interest we have for instance:

$$
\begin{aligned}
& D=11, N=1,128_{\text {boson }}+128_{\text {fermions }} \\
& (b=44+84, f=128) \\
& D=10, N=1 \text { (chiral) } \\
& N=1 \text { (matter) }\left(G=E_{8} \times E_{8}, \mathrm{SO}(32)\right) \\
& N=2 \text { : type A (non-chiral), } \\
& \text { type B (chiral) } \\
& D=4, N=1 \text { (chiral) : obtained as } M \text {-theory } \\
& \text { on } M_{7}=C Y_{3} \times S_{1} / Z_{2} \\
& N=8 \text { (non-chiral) }(b=56+70+2 \text {, } \\
& f=112+16) \text { : obtained as } \\
& M \text {-theory on } T_{7}\left(\mathrm{U}(1)^{28}\right. \text { gauge } \\
& \text { group) } \\
& \text { or on } S_{7}(\mathrm{SO}(8) \text { gauge group) . }
\end{aligned}
$$

Let us summarize some of the main basic results of the years $94-96$, in the context of string theory and its non-perturbative regime.

1) The Seiberg-Witten solution of rigid $N=2$ theory generalizes to heterotic-type II duality relating $K_{3} \times T_{2}$ vacua of heterotic to Calabi-Yau vacua of type II strings 30 31.

The second quantized mirror symmetry 30 gives exact non-perturbative results in $N=$ 2 superstrings, $D=4$. In particular, duality relates world-sheet instanton effects on the type II side tospace-time instantons on the heterotic side2232. Dual pair heterotic-type II theory constructions were proposed.

2) The implication of string-string duality in six dimensions for $S-T$ duality at $D=4$ was first shown by Duff 19 , and $U$-duality as a non-perturbative symmetry of different string theorjes was formulated by Hull and Townsend20.

3) Witten 21 proved the equivalence of different string theories in higher dimension and the duality of type IIA at strong coupling with $11 D$ supergravity at large radius ( $M$-theory on $\left.M_{10} \times S_{1}\right)$. Type $\amalg$ is self-dual at $D=$ $10(\mathrm{SL}(2, Z)$ duality $) 34$.

4) The $E_{8} \times E_{8}$ heterotic string at strong coupling is dual to the $M$-theory on $M_{10} \times S_{1} / Z_{2}$ (Horava-Witten) 33 .

5) The $\mathrm{SO}(32)$ Type I and $\mathrm{SO}(32)$ heterotic at $D=10$ are interchanged by weak-strong coupling duality (Polchinski-Witten) 35.

6) Open strings naturally arise, by the mechanism of tadpole cancellations, as sectors of type IIB closed strings on orientifolds 3637. Their end-points end on $D$-branes 38 , carrying $\mathrm{R}-\mathrm{R}$ charges. Phase transitions in six dimensions are possible 39 , and evidence for a nop-perturbative origin of gauge symmetries 40 was substantiated 41 .

7) $T$-duality at $D=9$ relates type IIA and type IIB theories, as well as $\mathrm{SO}(32)$ and $E_{8} \times$ $E_{8}$ heterotic strings in their broken phase $\mathrm{SO}(16) \times \mathrm{SO}(16) 35$.

8) $M$-theory and strings may undergo a further unification in twelve dimensions $(F$ theory) 27 .

New predictions of non-perturbative string theories can be derived from these nonperturbative relations between the five seemingly different superstring theories. As a circumstantial example 42 , strongly coupled heterotic string meets the agreement of $\alpha_{G U T}$ as measured (at LEP) from low-energy data.

In weakly coupled heterotic string, compactified on a Calabi-Yau threefold of size $V \approx M_{G U T}^{-6}$ with $G_{N}=\frac{e^{2 \phi}\left(\alpha^{\prime}\right)}{64 \pi V}$,

$$
\alpha_{G U T}=e^{2 \phi}\left(\alpha^{\prime}\right)^{3} / 16 \pi V \rightarrow G_{N}=\alpha_{G U T} \alpha^{\prime} / 4
$$

If $e^{2 \phi} \leq 1, G_{N} \geq \alpha_{G U T}^{4 / 3} / M_{G U T}^{2}$, which is too large compared to experiment. 
In type I string (weak coupling),

$$
\begin{aligned}
\alpha_{G U T} & =\frac{e^{\phi_{I}}\left(\alpha^{\prime}\right)^{3}}{16 \pi V}, G_{N}=\frac{e^{2 \phi_{I}}\left(\alpha^{\prime}\right)}{64 \pi V} \\
& \rightarrow G_{N}=e^{\phi_{I}} \alpha_{G U T} \alpha^{\prime} / 4
\end{aligned}
$$

Here, $G_{N}$ can be small.

In the $M$-theory set-up ( $\kappa$ is the $11 D$ gravitational coupling and $\rho$ the compactification radius)

$$
G_{N}=\frac{k^{2}}{16 \pi^{2} V \rho}, \quad \alpha_{G U T}=\frac{\left(4 \pi k^{2}\right)^{2 / 3}}{2 V} \ll 1 .
$$

So no disagreement with the experimental input exists in principle.

Finally, supersymmetry breaking can be described in a natural way both through a strongly coupled hidden gauge sector leading to gaugino condensation 43 and through the no-scale structure 44 of $M$-theory. The decompactification problem may be avoided 4547.

\section{Conclusions}

In the talk he gave at the Stpyy-Brook conference in 1979, Murray Gell-Mann 48 outlined the problems encountered with $N=8$ supergravity 19 :

1) "Predict a gauge group $\mathrm{SO}(8)$ that does not contain $\mathrm{SU}(3) \times \mathrm{SU}(2) \times \mathrm{U}(1)$;

2) 'Sign error' in the prediction of the cosmological constant $\lambda \approx M_{\text {Planck }}^{4}$ !

3) We can use lower- $N$ supersymmetric theories, but then we have a lack of unification ..."

It may be surprising to realize that the unphysical theory discussed by Gell-Mann 17 years ago is just a different vacuum of the very same theory whose dynamics hopefully encodes the standard model of our low-energy world!

\section{References}

1. For reviews on Supersymmetric Gauge Theories, see for instance:

J. Bagger and J. Wess, Supersymmetry and Supergravity (Princeton University Press, 1991);

S. Ferrara, Supersymmetry (North HollandWorld Scientific, 1987).
2. For a review on Superstrings, see: M. Green, J. Schwarz and E. Witten, Superstring Theory (Cambridge University Press, 1987).

3. J. Iliopoulos and B. Zumino, Nucl. Phys. B76 (1974) 310;

S. Ferrara, J. Iliopoulos and B. Zumino, Nucl. Phys. B77 (1974) 413;

M.T. Grisaru, W. Siegel and M. Roček, Nucl. Phys. B159 (1979) 429;

L. Girardello and M.T. Grisaru, Nucl. Phys. B194 (1982) 65.

4. N. Seiberg and E. Witten, Nucl. Phys. B426 (1994) 19 and B431 (1994) 484.

5. A. Klemm, W. Lerche, S. Theisen and S. Yankielowicz, Phys. Lett. B344 (1995) 169; P. Argyres and A. Faraggi, Phys. Rev. Lett. 74 (1995) 3931.

6. P.A.M. Dirac, Proc. Roy. Soc. A33 (1931) 60.

7. J. Schwinger, Science 165 (1969) 757;

D. Zwanziger, Phys. Rev. 176 (1968) 1489.

8. G. 't Hooft, Nucl. Phys. B79 (1974) 276; A.M. Polyakov, JETP. Lett. 20 (1974) 194.

9. E.B. Bogomolny, Sov. J. Nucl. Phys. 24 (1976) 449.

10. M.K. Prasad and C.M. Sommerfeld, Phys. Rev. Lett. 35 (1975) 760.

11. C. Montonen and D. Olive, Phys. Lett. 72B (1977) 117.

12. L. Girardello, A. Giveon, M. Porrati and A. Zaffaroni, Phys. Lett. B334 (1994) 331.

13. N. Seiberg, Phys. Lett. B206 (1988) 75.

14. R. Haag, J.T. Lopuszanski and M. Sohnius, Nucl. Phys. B88 (1975) 257.

15. E. Witten and D. Olive, Phys. Lett. 78B (1978) 97.

16. N. Seiberg, Phys. Rev. D49 (1994) 6857.

17. E. Cremmer, S. Ferrara and J. Scherk, Phys. Lett. 74B (1978) 61;

S. Ferrara, J. Scherk and B. Zumino, Nucl. Phys. B121 (1977) 393.

18. A. Sen, Int. J. Mod. Phys. A9 (1994) 3707; J.H. Schwarz, Lett. Math. Phys. 34 (1995) 309.

19. M. Duff, Nucl. Phys. B442 (1995) 47.

20. C. Hull and P. Townsend, Nucl. Phys. B438 (1995) 109.

21. E. Witten, Nucl. Phys. B443 (1995) 85.

22. A. Ceresole, R. D'Auria and S. Ferrara, Phys. Lett. 339B (1994) 71;

A. Ceresole, R. D'auria, S. Ferrara and A. 
van Proeyen, Nucl. Phys. B444 (1995) 92.

23. S. Kachru, A. Klemm, W. Lerche, P. Mayr and C. Vafa, Nucl. Phys. B459 (1996) 537.

24. M.J. Duff and J.X. Lu, Nucl. Phys. B426 (1994) 301.

25. E. Witten, preprint IASSNS-HEP-9563 (hep-th/9507121), contribution to Strings '95, Los Angeles, 1995.

26. E. Cremmer, B. Julia and J. Scherk, Phys. Lett. 76B (1978) 409.

27. C. Vafa, Nucl. Phys. B469 (1996) 403.

28. A. Salam and E. Sezgin, Supergravity in diverse dimensions (North-Holland-World Scientific, 1989).

29. W. Nahm, Nucl. Phys. B135 (178) 149.

30. S. Ferrara, J. Harvey, A. Strominger and C. Vafa, Phys. Lett. B361 (1995) 59.

31. S. Kachru and C. Vafa, Nucl. Phys. B450 (1995) 69.

32. A. Strominger, Nucl. Phys. B451 (1995) 97;

B. Greene, D. Morrison and A. Strominger, Nucl. Phys. 451 (1995) 109.

33. P. Horava and E. Witten, Nucl. Phys. B460 (1996) 506.

34. For recent reviews. see:

J. Schwarz, preprint CALTECH-68-2065 (hep-th/9607201);

A. Sen, preprint MRI-PHY-96-28 hepth/9609176);

M. Duff, preprint CPT-TAMU-33-96 hepth/9608117).

35. J. Polchinski and E. Witten, Nucl. Phys. B460 (1996) 525.

36. A. Sagnotti, Open Strings and their Symmetry Groups, talk at the Cargèse Summer Institute (1987);

G. Pradisi and A. Sagnotti, Phys. Lett. B216 (1989) 59;

M. Bianchi, G. Pradisi and A. Sagnotti, Nucl. Phys. B376 (1992) 365.

37. J. Dai, R. Leigh and J. Polchinski, Mod. Phys. Lett. A4 (1989) 2073;

P. Horava, Nucl. Phys. B227 (1989) 461;

J. Polchinski, Phys. Rev. D50 (1994) 6041.

38. J. Polchinski, Phys. Rev. Lett. 75 (1995) 4734 .

39. A. Sagnotti, Phys. Lett. 294B (1992) 196.

40. E. Witten, Nucl. Phys. B471 (1996) 121.

41. M. Duff, R. Minasian and E. Witten, Nucl. Phys. B465 (1996) 44.
42. E. Witten, Nucl. Phys. B471 (1996) 121.

43. P. Horava, preprint PUPT-1637 hepth/9608019).

44. E. Cremmer, S. Ferrara, C. Kounnas and D.V. Nanopoulos, Phys. Lett. B133 (1983) 61 ;

J. Ellis, A. Lahanas, D.V. Nanopoulos and K. Tamvakis, Phys. Lett. B134 (1984) 419; J. Ellis, C. Kounnas and D.V. Nanopoulos, B241 (1984) 406.

45. T. Banks and M. Dine, preprint SCIPP96-26 (hep-th/9609046); preprint RU-96-27 (hep-th/9605136).

46. E. Kiritsis, C. Kounnas, P.M. Petropoulos and J. Rizos, preprint CERN-TH/96-90 (hep-th/9608034).

47. I. Antoniadis and M. Quiros, CPTH-S4650996 (hep-th/9609209).

48. M. Gell-Mann in Supergravity, eds. D.Z. Freedman and P. van Nieuwenhuizen (North-Holland, Amsterdam, 1979), p. 315.

49. J. Cremmer and B. Julia, Nucl. Phys. B159 (1979) 141;

B. de Wit and H. Nicolai, Nucl. Phys. B208 (1982) 323.

50. L. Alvarez-Gaumé, J. Distler, C. Kounnas and M. Mariño, Int. J. Mod. Phys. A11 (1996) 4745.

\section{Questions}

\section{G.G. Ross, Oxford University:}

What progress has there been towards breaking supersymmetry and using duality to give information about normal QCD?

\section{S. Ferrara:}

Alvarez-Gaumé, Distler, Kounnas and Mariño 5 have analysed soft breaking terms preserving the analyticity properties of the SeibergWitten solution. This allows a detailed description of the onset of the confinement transition and the pattern of chiral symmetry breaking. When those results are extrapolated to a limit where supersymmetry decouples and then QCD is retrieved, an indication that the $\mathrm{QCD}$ vacuum may require the simultaneous occurrence of mutually non-local degrees of freedom (monopoles and dyons) seems to emerge.

G. Veneziano, CERN: In order to get a successful 
relation between the Planck scale and the GUT scale is it crucial that the original type II theory is strongly coupled or can the coupling be just at the self-dual value $=1$ ?

\section{S. Ferrara:}

The strong coupling in heterotic theory means that its dual theory is weakly coupled. Therefore the self-dual value seems not appropriate for this regime. 